\title{
ON GENERALIZED LOWER(K)RECORD VALUES FROM THE FRÉCHET DISTRIBUTION
}

\author{
P. Yageen Thomas* and Jerin Paul*
}

\begin{abstract}
In this paper we study the generalized lower(k)record values arising from the Fréchet distribution. Expressions for the moments and product moments of those generalized lower(k)record values are derived. Some properties of generalized lower(k) record values which characterize the Fréchet distribution have been established. Also some distributional properties of generalized lower(k)record values arising from the Fréchet distribution are considered and used for suggesting an estimator for the shape parameter of the Fréchet distribution. The location and scale parameters are estimated using the Best Linear Unbiased Estimation procedure. Prediction of a future record using the Best Linear Unbiased Predictor has been studied. A real life data set is used to illustrate the results generated in this work.
\end{abstract}

Key words and phrases: Best Linear Unbiased Estimation, Best Linear Unbiased Predictor, characterizations, Fréchet distribution, generalized lower(k)record values.

\section{Introduction}

Record values and associated statistics are of great importance in several real life problems involving weather, economic studies, sports and so on. Chandler (1952) defined the record statistics as successive extremes occurring in a sequence of independent and identically distributed (iid) random variables. The prediction of a future record value is an interesting problem with many real life applications. For example the predicted value of the amount of the next record level of water that a dam will capture and hold or discharge is helpful for future planning purposes, predicted intensity of the next strongest earthquake is essential for disaster management planning, prediction of the next level of a new record in athletic events is helpful for subjecting the prospective athletes to rigorous training and practice and so on.

In the available literature, much thrust is seen given to upper record values of sequences of iid observations drawn from continuous distributions. Lower record values have their own importance in certain problems of study. For example in stock markets a lower record value of the market index is of much interest to prospective investors to plan for their investment strategies. Lower record values of time to cover a fixed distance in freestyle swimming or in a marathon walk are interesting information for coaches participants and observers. For more details on the applications of record values, see Ahsanullah (1998), Arnold et al. (1998) and Nevzorov (2001).

Usually in many events such as athletics, share prices, temperatures etc.

\footnotetext{
Received April 7, 2014. Revised August 8, 2014. Accepted August 11, 2014.

*Department of Statistics, University of Kerala, Kariavattom, Trivandrum, India. Email: yageenthomas@gmail.com
} 
interest lies on record values only and they alone are stored for future reference. In such situations one is compelled to rely only upon the available record data to deal with inference problems of the parent distribution. A difficulty that one encounters in dealing with statistical inference problems based on record values has to do with the limited occurrence of record data, as the expected waiting time of occurrence of every record after the first is infinite. However one may observe that generally the $k$ th record values as introduced by Dziubdziela and Kopocinski (1976) occur more frequently than those of the classical records. Further the sequence of $k$ th record values is free from the inclusion of outliers occurring in the data. Suppose $\left\{X_{n}\right\}$ is a sequence of iid random variables. Then for a positive integer $k \geq 1$, the sequence of $k$ th lower record times $\left\{T_{L(n, k)}, n \geq 1\right\}$ is defined as follows:

$$
T_{L(1, k)}=1, \quad \text { with probability } 1
$$

and for $n>1$,

$$
T_{L(n, k)}=\min \left\{j: j>T_{L(n-1, k)}, X_{k: T_{L(n-1, k)+k-1}}>X_{k: j+k-1}\right\},
$$

where $X_{i: m}$ denotes the $i$ th order statistic in a sample of size $m$. Now if we write

$$
X_{L(n, k)}=X_{k: T_{L(n-1, k)}+k-1}, \quad \text { for } \quad n=1,2, \ldots,
$$

then $\left\{X_{L(n, k)}\right\}$ is known as the sequence of the $k$ th lower record values. For similar definitions of the $k$ th upper record times and $k$ th upper record values see, Bieniek and Szynal (2007) and Nevzorov (2001, p. 82). If the parent distribution is absolutely continuous with cumulative distribution function (cdf) $F(x)$ and probability density function (pdf) $f(x)$, then the pdf $f_{X_{L(n, k)}}(x)$ of $X_{L(n, k)}$ and the joint pdf $f_{X_{L(m, k)}, X_{L(n, k)}}(x, y)$ of $X_{L(m, k)}$ and $X_{L(n, k)}$ are given by (see, Pawlas and Szynal (1998, 2000))

$$
f_{X_{L(n, k)}}(x)=\frac{k^{n}}{\Gamma(n)}[-\ln \{F(x)\}]^{n-1}[F(x)]^{k-1} f(x), \quad-\infty<x<\infty,
$$

and

$$
\begin{aligned}
f_{X_{L(m, k)}, X_{L(n, k)}}(x, y)= & \frac{k^{n}[-\ln \{F(x)\}]^{m-1}[\ln \{F(x)\}-\ln \{F(y)\}]^{n-m-1}}{\Gamma(m) \Gamma(n-m)} \\
& \times[F(y)]^{k-1} \frac{f(x) f(y)}{F(x)}
\end{aligned}
$$

where $x>y, 1 \leq m<n, n \geq 2$.

The $k$ th member of the sequence of the classical record values is also called as $k$ th record value. This contradicts the $k$ th record values as defined in Dziubdziela and Kopocinski (1976). Pointing out this conflict in the usage of the $k$ th record values of Dziubdziela and Kopocinski (1976), and as it generates the classical record values for $k=1$, Minimol and Thomas $(2013,2014)$ have called the $k$ th record values as defined in Dziubdziela and Kopocinski (1976) the Generalized record values. Agreeing with the contention of Minimol and Thomas (2013, 
2014), we also call the $k$ th record values of Dziubdziela and Kopocinski (1976) generalized(k)record values all through this paper.

It is to be noted that a lot of research is going on to detect outliers in data so as to delete them and devise more reasonable statistical methods to the problem of interest. The integer parameter $k$ involved in generalized lower(k)record value (GLRV) can be chosen such that the type of record data that we have to depend upon discards the possible outliers which creep into the data. For example if initial scrutiny of the data reveals that there is a possibility of the occurrence of only one outlier in terms of its smallness in magnitude in the data, then it is enough to consider generalized lower(2)record values as the desirable record data that may be used for further analysis and storage of it for future purposes.

Inventing more and more characterization results based on the distributional properties of the statistics arising from a distribution makes the model mathematically tractable for developing statistical methods to analyse the data arising from it. Characterization properties makes a distribution mathematically informative and help a practicing statistician to decide on the suitability of the model in proposing it to a population from which some data sets are available. More over characterization properties based on generalized(k)record values for $k>1$ makes the distribution capable of dealing with the analysis of the record data by eliminating some possible outliers which creep into the data. The above considerations motivate the authors to make a study on GLRVs arising from the well-known Fréchet distribution (FD).

Several applications of GLRVs can be found in the literature. For some recent works on characterization of parent distributions using recurrence relations on moments of generalized record values see, Minimol and Thomas $(2013,2014)$ and Pawlas and Szynal (1998, 1999, 2000).

In this work we mainly deal with some interesting properties of GLRVs arising from the FD and the application of those record values to deal with some inference problems of this distribution. Section 2 deals with the review of the FD and describes an instance where this distribution is found to be a suitable model. In Section 3 we derive the exact expressions for the means, variances and covariances of GLRVs arising from the FD. Further we have identified certain properties of GLRVs which characterize the FD and those characterization theorems are given in Section 4. In Section 5 we derive some interesting distributional aspects of the GLRV arising from the FD. Section 6 deals with the problem of estimation of the location and scale parameters of the FD when the shape parameter involved in it is known. In this section we discuss the simultaneous estimation of all parameters of the FD as well. In the last Section we consider the problem of prediction of the next immediate future GLRV that may occur from the FD using a Best Linear Unbiased Predictor (BLUP) based on initial GLRVs arising from a FD. We have illustrated further the method of predicting the next GLRV based on the initial GLRVs arising from the FD by using real life data. 


\section{Fréchet distribution}

A random variable $Z$ is said to have a FD (see, Fréchet (1927)) if its probability density function is given by

$$
\begin{aligned}
& g(z)=\frac{c}{\sigma}\left(\frac{z-\theta}{\sigma}\right)^{-c-1} \exp \left\{-\left(\frac{z-\theta}{\sigma}\right)^{-c}\right\}, \\
& z>\theta, \sigma>0,-\infty<\theta<\infty, \quad c>0,
\end{aligned}
$$

where $\theta, \sigma$ and $c$ are known as location, scale and shape parameters respectively. The cdf corresponding to the pdf given in (2.1) is

$$
G(z)=\exp \left\{-\left(\frac{z-\theta}{\sigma}\right)^{-c}\right\}, \quad z>\theta, \sigma>0,-\infty<\theta<\infty, c>0 .
$$

The standard form of the FD is one which is free from the location parameter $\theta$ and scale parameter $\sigma$ and is given by the pdf

$$
f_{0}(y)=c y^{-c-1} e^{-y^{-c}}, \quad y>0, c>0
$$

with the corresponding cdf given by

$$
F_{0}(y)=e^{-y^{-c}}, \quad y>0, c>0 .
$$

The FD (also known as inverse Weibull distribution) as defined in (2.1) can also be realized to be a special case of the generalized extreme value distribution (for details see, Castillo et al. (2004, p. 63)). Further works on this distribution are available in Bury (1999), Coles et al. (2001) and Castillo et al. (2004). Attempts at estimating the parameters using classical record values arising from (2.1) are seen in Sultan (2010).

The FD is considered to be a very good model to analyze data sets dealing with yearly sports and athletic records maintained over a period of time. For example consider the following data as reported in http://www.olympicsrecords.com regarding the Olympic records on time taken to cover the $200 \mathrm{~m}$ men's freestyle swim during the years 1900 to 2012.

If we apply the maximum likelihood method of estimation using Mathematica software, the estimates of the parameters obtained are given by $c=1.33694$, $\sigma=5.3266$ and $\theta=100.825$. The value of the Kolmogrov-Smirnov statistic computed for assessing the goodness-of-fit is 0.13023 which lies far below the $5 \%$ table critical value of 0.34890 . Thus we conclude that the FD as defined in (2.1) is a good fit to the yearly record data on time taken to cover $200 \mathrm{~m}$ in the men's freestyle swim.

Table 1. Olympics-Men's 200 meter freestyle swim.

\begin{tabular}{|c|c|c|c|c|c|c|c|c|c|}
\hline Year & Time & Year & Time & Year & Time & Year & Time & Year & Time \\
\hline 1900 & 145.02 & 1972 & 112.78 & 1984 & 107.44 & 1996 & 107.63 & 2008 & 102.96 \\
1904 & 164.02 & 1976 & 110.29 & 1988 & 107.25 & 2000 & 105.35 & 2012 & 103.14 \\
1968 & 115.02 & 1980 & 109.81 & 1992 & 106.7 & 2004 & 104.71 & & \\
\hline
\end{tabular}




\section{GLRV arising from standard Fréchet distribution}

Let $\left\{Y_{L(i, k)}\right\}$ be the sequence of GLRVs arising from the standard FD defined in (2.3). Then by (1.1) the pdf of $Y_{L(n, k)}$ is given by

$$
f_{Y_{L(n, k)}}(x)=\frac{k^{n} c}{\Gamma(n)} x^{-c n-1} e^{-k x^{-c}}, \quad n=1,2, \ldots, k=1,2, \ldots, c>0 .
$$

The joint pdf of $Y_{L(m, k)}$ and $Y_{L(n, k)}$ for $m<n$ based on (1.2) is given by

$$
\begin{aligned}
& f_{Y_{L(m, k)}, Y_{L(n, k)}}(x, y) \\
& \quad=\frac{c^{2} k^{n}}{\Gamma(m) \Gamma(n-m)}\left(y^{-c}-x^{-c}\right)^{n-m-1} x^{-c m-1} y^{-c-1} e^{-k y^{-c}},
\end{aligned}
$$

where $0<y<x<\infty$. Using the expressions given in (3.1) and (3.2), we have derived the exact expressions for the means, variances and covariances of GLRVs arising from the standard FD and they are given below as:

$$
\begin{aligned}
E\left(Y_{L(n, k)}^{i}\right)= & k^{i / c} \frac{\Gamma\left(n-\frac{i}{c}\right)}{\Gamma(n)}, \quad \text { provided } n>\frac{i}{c}, \\
\operatorname{Var}\left(Y_{L(n, k)}\right) & =\operatorname{Cov}\left(Y_{L(n, k)}, Y_{L(n, k)}\right) \\
& =\frac{k^{2 / c}}{\Gamma(n)}\left\{\Gamma\left(n-\frac{2}{c}\right)-\frac{\left(\Gamma\left(n-\frac{1}{c}\right)\right)^{2}}{\Gamma(n)}\right\},
\end{aligned}
$$

provided $n>\frac{2}{c}$, and

$$
\begin{aligned}
\operatorname{Cov}\left(Y_{L(m, k)}, Y_{L(n, k)}\right) & \\
= & \frac{k^{2 / c} \Gamma\left(m-\frac{1}{c}\right)}{\Gamma(m)} \\
& \times\left\{\frac{\Gamma\left(n-\frac{2}{c}\right)}{\Gamma\left(n-\frac{1}{c}\right)}-\frac{\Gamma\left(n-\frac{1}{c}\right)}{\Gamma(n)}\right\}, \quad \text { provided } n>\frac{2}{c} .
\end{aligned}
$$

\section{Characterization of Fréchet distribution using GLRV}

In this section we deal with some properties of GLRVs which characterize a sub-family $\mathscr{F}$ of distributions belonging to the general family of the FD defined by the pdf (2.1). We say that a random variable $X$ has a distribution belonging to $\mathscr{F}$ if its pdf is given by

$$
f(x)=\frac{c}{\sigma}\left(\frac{x}{\sigma}\right)^{-c-1} e^{-(x / \sigma)^{-c}}, \quad x>0, \sigma>0 .
$$


The cdf corresponding to (4.1) is then defined by

$$
F(x)=e^{-(x / \sigma)^{-c}}, \quad x>0, \sigma>0 .
$$

Throughout our subsequent discussions we may write $\bar{F}(x)=1-F(x)$ to denote the survival function corresponding to the $\operatorname{cdf} F(x)$. We now prove the following Theorem.

THEOREM 1. Let $\left\{X_{n}, n \geq 1\right\}$ be a sequence of iid random variables each distributed identically with a common continuous cdf $F(x)$ and pdf $f(x)$. Let $\bar{F}(0)=1-F(0)=1$ and $F(x)<1$ for all $x>0$. Let $\left\{X_{L(n, k)}\right\}$ be the sequence of GLRVs generated from the sequence $\left\{X_{n}\right\}$. Then $F(x)=e^{-(x / \sigma)^{-c}}$ for all $x>0, c>0, \sigma>0$, if and only if $T=\frac{X_{L(n, k)}}{X_{L(m, k)}}$ and $U=X_{L(n, k)},($ for $m<n)$ are independently distributed.

Proof. The joint pdf of $X_{L(m, k)}$ and $X_{L(n, k)}$, is given by

$$
\begin{aligned}
& f_{X_{L(m, k)}, X_{L(n, k)}}(x, y) \\
& \quad=\frac{k^{n}}{\Gamma(m) \Gamma(n-m)}[H(x)]^{m-1}[H(y)-H(x)]^{n-m-1}[F(y)]^{k} h(x) h(y),
\end{aligned}
$$

where $H(x)=-\ln F(x)$ and $h(x)=\frac{d}{d x} H(x), 0<y<x<\infty$. If $F(x)=$ $e^{-(x / \sigma)^{-c}}$ for every $x>0, \sigma>0$ and $c>0$, then the joint pdf $f_{X_{L(m, k)}, X_{L(n, k)}}(x, y)$ of $X_{L(m, k)}$ and $X_{L(n, k)}$ is given by

$$
\begin{aligned}
& f_{X_{L(m, k)}, X_{L(n, k)}}(x, y) \\
& \quad=\frac{k^{n} c^{2} \sigma^{c n}}{\Gamma(m) \Gamma(n-m)}\left\{y^{-c}-x^{-c}\right\}^{n-m-1} x^{-c m-1} y^{-c-1} e^{-k(y / \sigma)^{-c}} .
\end{aligned}
$$

Consider the transformations $T=\frac{X_{L(n, k)}}{X_{L(m, k)}}$ and $U=X_{L(n, k)}$. Then the Jacobian of the transformation is $|J|=\frac{u}{t^{2}}$. Thus we can write the joint pdf $f_{T, U}(t, u)$ of $T$ and $U$ as

$$
f_{T, U}(t, u)=\frac{c^{2} k^{n} \sigma^{c n}}{\Gamma(m) \Gamma(n-m)}\left(1-t^{c}\right)^{n-m-1} u^{-c n-2} t^{c m+1} e^{-k(u / \sigma)^{-c}} \frac{u}{t^{2}},
$$

where $0<t<1, u>0, \sigma>0$ and $c>0$. The marginal pdf of $T$ is given by

$$
\begin{aligned}
f_{T}(t) & =\frac{c^{2} k^{n} \sigma^{c n}}{\Gamma(m) \Gamma(n-m)} \int_{0}^{\infty}\left(1-t^{c}\right)^{n-m-1} u^{-c n-2} t^{c m+1} e^{-k(u / \sigma)^{-c}} \frac{u}{t^{2}} \mathrm{~d} u \\
& =\frac{c \Gamma(n)}{\Gamma(m) \Gamma(n-m)}\left(1-t^{c}\right)^{n-m-1} t^{c m-1}
\end{aligned}
$$

Also the pdf $f_{U}(u)$ of $U$ is given by

$$
f_{U}(u)=\frac{k^{n} c}{\sigma \Gamma(n)}\left(\frac{u}{\sigma}\right)^{-c n-1} e^{-k(u / \sigma)^{-c}}
$$


From (4.5), (4.6) and (4.7) we obtain $f_{T, U}(t, u)=f_{T}(t) f_{U}(u)$. Hence $T$ and $U$ are independently distributed.

Now we prove the sufficient part of the Theorem. Let $T=\frac{X_{L(n, k)}}{X_{L(m, k)}}$ and $U=$ $X_{L(n, k)}$ be distributed independently. Then the Jacobian of the transformation is $|J|=\frac{u}{t^{2}}$. Thus using (1.2) we can write the joint pdf $f_{T, U}(t, u)$ of $T$ and $U$ as

$$
\begin{aligned}
f_{T, U}(t, u)= & \frac{k^{n}}{\Gamma(m) \Gamma(n-m)}\left[-\ln F\left(\frac{u}{t}\right)\right]^{m-1}\left[\ln F\left(\frac{u}{t}\right)-\ln F(u)\right]^{n-m-1} \\
& \times[F(u)]^{k} \frac{f\left(\frac{u}{t}\right)}{F\left(\frac{u}{t}\right)} \frac{f(u)}{F(u)} \frac{u}{t^{2}} .
\end{aligned}
$$

We replace $-\ln F\left(\frac{u}{t}\right)$ by $H\left(\frac{u}{t}\right)$ and $-\ln F(u)$ by $H(u)$. Hence their derivatives are $h\left(\frac{u}{t}\right)=\frac{f\left(\frac{u}{t}\right)}{F\left(\frac{u}{t}\right)} \frac{u}{t^{2}}$ and $h(u)=-\frac{f(u)}{F(u)}$. Therefore

$$
\begin{aligned}
f_{T, U}(t, u)= & \frac{k^{n}}{\Gamma(m) \Gamma(n-m)}\left[H\left(\frac{u}{t}\right)\right]^{m-1}\left[H(u)-H\left(\frac{u}{t}\right)\right]^{n-m-1} \\
& \times[F(u)]^{k} h\left(\frac{u}{t}\right)[-h(u)] .
\end{aligned}
$$

The pdf $f_{U}(u)$ of $U$ is given by

$$
f_{U}(u)=\frac{k^{n}}{\Gamma(n)}[H(u)]^{n-1}[F(u)]^{k}[-h(u)] .
$$

Since $T$ and $U$ are independent, we get the pdf $f_{T}(t)$ of $T$ from (4.8) and (4.9) as

$$
f_{T}(t)=\frac{\Gamma(n)}{\Gamma(m) \Gamma(n-m)}\left[\frac{H\left(\frac{u}{t}\right)}{H(u)}\right]^{m-1}\left[1-\frac{H\left(\frac{u}{t}\right)}{H(u)}\right]^{n-m-1} \frac{h\left(\frac{u}{t}\right)}{H(u)} .
$$

The distribution function of $T$ is given by

$$
\begin{aligned}
F_{T}(t)= & \frac{\Gamma(n)}{\Gamma(m) \Gamma(n-m)} \\
& \times \int_{0}^{t}\left[\frac{H\left(\frac{u}{v}\right)}{H(u)}\right]^{m-1}\left[1-\frac{H\left(\frac{u}{v}\right)}{H(u)}\right]^{n-m-1} \frac{\partial}{\partial v}\left[\frac{H\left(\frac{u}{v}\right)}{H(u)}\right] \mathrm{d} v .
\end{aligned}
$$

On putting $\frac{H(u / v)}{H(u)}=z$, we obtain

$$
F_{T}(t)=\frac{\Gamma(n)}{\Gamma(m) \Gamma(n-m)} \int_{0}^{H(u / t) / H(u)} z^{m-1}(1-z)^{n-m-1} \mathrm{~d} z .
$$


We then have

$$
F_{T}(t)=\sum_{r=m}^{n-1}\left(\begin{array}{c}
n-1 \\
r
\end{array}\right)\left[\frac{H\left(\frac{u}{t}\right)}{H(u)}\right]^{r}\left[1-\frac{H\left(\frac{u}{t}\right)}{H(u)}\right]^{n-r-1}
$$

The above result is true for every value of $m$, for $m<n$. On putting $m=n-1$. We then have

$$
F_{T}(t)=\left[\frac{H\left(\frac{u}{t}\right)}{H(u)}\right]^{n-1}
$$

That is,

$$
\left[H\left(\frac{u}{t}\right)\right]^{n-1}=F_{T}(t)[H(u)]^{n-1}
$$

where $F_{T}(t)$ is a function of $t$ alone. Since $0<u<\infty$, on putting $u=1$, in the above equation we get

$$
\left[\lambda H\left(\frac{1}{t}\right)\right]^{n-1}=F_{T}(t),
$$

where $\lambda=[H(1)]^{-1}>0$ is a constant. Thus we have

$$
\left[H\left(\frac{u}{t}\right)\right]^{n-1}=\left[\lambda H\left(\frac{1}{t}\right)\right]^{n-1}[H(u)]^{n-1} \quad \text { for } \quad 0<t<1 .
$$

Hence on multiplying both sides of the above equation by $\lambda^{n-1}$ we get

$$
\begin{aligned}
& {\left[\lambda H\left(\frac{u}{t}\right)\right]^{n-1}=\left[\lambda H\left(\frac{1}{t}\right)\right]^{n-1}[\lambda H(z)]^{n-1} \text { and hence we write }} \\
& {\left[\lambda H\left(\frac{u}{t}\right)\right]=\left[\lambda H\left(\frac{1}{t}\right)\right][\lambda H(z)] .}
\end{aligned}
$$

The above equation can be further written as

$$
\left[H_{1}(w u)\right]=\left[H_{1}(w)\right]\left[H_{1}(z)\right]
$$

where, $H_{1}(\cdot)=\lambda H(\cdot)$. From Aczel (1960), we observe that the only continuous solution of (4.11) is $H_{1}(x)=x^{d}$, for all $x>0$ where $d$ is a constant. Thus on writing $\lambda=\sigma^{d}$ we have $F(x)=e^{-(x / \sigma)^{d}}$. As $F(x)$ is a distribution function with $\bar{F}(0)=1, d$ must be negative. Hence we write $F(x)=e^{-(x / \sigma)^{-c}}$, where the constant $c$ is positive. This completes the proof.

One can also state some more results whose proofs are just similar to the proof of Theorem 1 and hence we state those results without proof in the following corollaries. 
Corollary 1. Let $\left\{X_{n}, n \geq 1\right\}$ be a sequence of iid random variables each distributed identically with a common continuous cdf $F(x)$ and $p d f f(x)$. Let $\bar{F}(0)=1$ and $F(x)<1$ for all $x>0$. Let $\left\{X_{L(n, k)}\right\}$ be the sequence of GLRVs generated from the sequence $\left\{X_{n}\right\}$. Then $F(y)=e^{-(x / \sigma)^{-c}}$ for all $x>0, \sigma>0$, $c>0$, if and only if $\frac{X_{L(n, k)}}{X_{L(m, k)}+X_{L(n, k)}}$ and $X_{L(n, k)}($ for $m<n)$ are independently distributed.

Corollary 2. Let $\left\{X_{n}, n \geq 1\right\}$ be a sequence of iid random variables each distributed identically with a common continuous cdf $F(x)$ and $p d f f(y)$. Let $\bar{F}(0)=1$ and $F(x)<1$ for all $x>0$. Let $\left\{X_{L(n, k)}\right\}$ be the sequence of GLRVs generated from the sequence $\left\{X_{n}\right\}$. Then $F(x)=e^{-(x / \sigma)^{-c}}$ for all $x>0, \sigma>0$, $c>0$, if and only if $\frac{X_{L(m, k)}}{X_{L(m, k)}-X_{L(n, k)}}$ and $X_{L(n, k)}($ for $m<n)$ are independently distributed.

Corollary 3. Let $\left\{X_{n}, n \geq 1\right\}$ be a sequence of iid random variables each distributed identically with a common continuous cdf $F(x)$ and $p d f f(x)$. Let $\bar{F}(0)=1$ and $F(x)<1$ for all $x>0$. Let $\left\{X_{L(n, k)}\right\}$ be the sequence of GLRVs generated from the sequence $\left\{X_{n}\right\}$. Then $F(x)=e^{-(x / \sigma)^{-c}}$ for all $x>0, \sigma>0$, $c>0$, if and only if $\frac{X_{L(n, k)}}{X_{L(m, k)}-X_{L(n, k)}}$ and $X_{L(n, k)}($ for $m<n)$ are independently distributed.

Corollary 4. Let $\left\{X_{n}, n \geq 1\right\}$ be a sequence of iid random variables each distributed identically with a common continuous cdf $F(x)$ and pdf $f(x)$. Let $\bar{F}(0)=1$ and $F(x)<1$ for all $x>0$. Let $\left\{X_{L(n, k)}\right\}$ be the sequence of GLRVs generated from the sequence $\left\{X_{n}\right\}$. Then $F(x)=e^{-(x / \sigma)^{-c}}$ for all $x>0, \sigma>0$, $c>0$, if and only if $\frac{X_{L(m, k)}+X_{L(n, k)}}{X_{L(m, k)}}$ and $X_{L(n, k)}($ for $m<n)$ are independently distributed.

Note 1. If we have several data sets from a population, then for any fixed $n$ and $m$ we may plot the points $(T, U)$ (where $T, U$ are variables as defined in Theorem 1) corresponding to each data set in a graph sheet. If the points in the graph sheet are seen scattered all over the region without any pattern then as an implication of Theorem 1, one may conclude that the FD as defined in (4.1) could be taken as a suitable distribution of the population random variable. Similar attempts may be made with the results in each of the Corollaries 1 to 4 to check the suitability of the FD as a model to the population distribution.

\section{Some distributional aspects of GLRV arising from the standard FD}

It is to be noted that the basic results that we require to establish some properties on GLRVs arising from a distribution are already seen derived for the 
equivalent situations on generalized upper(k)record value (GURV) (for details see, Stam (1985), Nevzorov (2001), Ahmadi et al. (2009)). Suppose $\left\{Y_{n}\right\}$ is a sequence of iid random variables. Then for a positive integer $k \geq 1$, the sequence of generalized upper $(\mathrm{k})$ record times $\left\{T_{U(n, k)}, n \geq 1\right\}$ is defined as (see, Nevzorov (2001, p. 82)):

$$
T_{U(1, k)}=k, \quad \text { with probability } 1
$$

and, for $n \geq 1$

$$
T_{U(n+1, k)}=\min \left\{j: j>T_{U(n, k)}, X_{j}>X_{T_{U(n, k)}-k+1: T_{U(n, k)}}\right\},
$$

where $Y_{i: m}$ denotes the $i$ th order statistic in a sample of size $m$. Now if we write

$$
Y_{U(n, k)}=Y_{T_{U(n, k)}-k+1: T_{U(n, k)}}, \quad \text { for } \quad n=1,2, \ldots,
$$

then $\left\{Y_{U(n, k)}\right\}$ is known as the sequence of the GURVs.

To derive the results to be discussed in this section we require some basic results such as the joint distribution of a collection of selected GLRVs arising from an arbitrary distribution whose support set is restricted to the set of positive real's say $\mathbb{R}^{+}$. It is clear to note that if $X$ is a random variable with cdf $F(x)$ and pdf $f(x)$ with the support $\mathbb{R}^{+}$and if $\left\{X_{j}, j \geq 1\right\}$ is a sequence of iid random variables which are identically distributed as $X$ then we may construct the sequence $\left\{X_{L(n, k)}, n \geq 1\right\}$ of the GLRVs of $\left\{X_{j}, j \geq 1\right\}$. In this case the sequence $\left\{Y_{n}\right\}$, where $Y_{n}=\frac{1}{X_{L(n, k)}}, n=1,2, \ldots$ turns out to be the sequence of GURVs arising from the sequence $\left\{\frac{1}{X_{j}}, j \geq 1\right\}$. In other words $Y_{n}, n=1,2, \ldots$ are distributed as the GURVs arising from a sequence of iid random variables each distributed identically with cdf $1-F\left(\frac{1}{y}\right)$ and pdf $\frac{1}{y^{2}} f\left(\frac{1}{y}\right)$ with the same support set $\mathbb{R}^{+}$. Most of the theories necessary to carry out the analysis based on GURVs are seen available in the existing literature (Nevzorov (2001), Ahmadi et al. (2009)).

Stam (1985) has established an excellent basic result which states that the Poisson processes generated by GURVs and the inter-record times are independent. Based on this property he has obtained the joint pdf of the first $n$ GURVs arising from any arbitrary distribution. If $\left\{X_{U(j, k)}, j=1,2, \ldots, n\right\}$ are the first $n$ GURVs from the basic distribution with pdf $f(x)$ and cdf $F(x)$ then from Stam (1985) the joint pdf of $\left\{X_{U(j, k)}, j=1,2, \ldots, n\right\}$ is given below

$$
\begin{aligned}
& f_{X_{U(1, k)}, X_{U(2, k)}, \ldots, X_{U(n, k)}}\left(x_{1}, x_{2}, \ldots, x_{n}\right) \\
& \quad=k^{n}\left[1-F\left(x_{n}\right)\right]^{k-1} f\left(x_{n}\right) \prod_{j=1}^{n-1} \frac{f\left(x_{j}\right)}{1-F\left(x_{j}\right)} .
\end{aligned}
$$

One may similarly establish the independence property of the Poisson processes generated by GLRVs and the associated inter-record times and thereby derive the joint pdf of the first $n$ GLRVs. However since our interest is with GLRVs arising from an arbitrary distribution with support set $\mathbb{R}^{+}$, we deduce 
those results from the existing results available on the GURVs $Y_{j}=\frac{1}{X_{L(j, k)}}$, $j=1,2, \ldots$ arising from a distribution in pdf $\frac{1}{y^{2}} f\left(\frac{1}{y}\right)$. In particular if we are interested in the joint pdf of $X_{L\left(r_{1}, k\right)}, X_{L\left(r_{2}, k\right)}, \ldots, X_{L\left(r_{i}, k\right)}$, where $r_{1}, r_{2}, \ldots, r_{i}$ are integers such that $1 \leq r_{1}<r_{2}<\cdots<r_{i} \leq n$, then we derive the joint pdf of $\frac{1}{X_{L\left(r_{1}, k\right)}}, \frac{1}{X_{L\left(r_{2}, k\right)}}, \ldots, \frac{1}{X_{L\left(r_{i}, k\right)}}$ using the result of Stam (1985) and then obtain the joint pdf of $X_{L\left(r_{1}, k\right)}, X_{L\left(r_{2}, k\right)}, \ldots, X_{L\left(r_{i}, k\right)}$ by an appropriate inverse transform. Thus we have the following lemma.

Lemma 1. Let $\left\{X_{j}, j \geq 1\right\}$ be a sequence of iid random variables with $p d f$ $f(x)$ and cdf $F(x)$ with support set $\mathbb{R}^{+}$. Let $\left\{X_{L(n, k)}\right\}$ be the sequence of GLRVs generated from the sequence $\left\{X_{j}, j \geq 1\right\}$. Let $r_{1}, r_{2}, \ldots, r_{i}$ be positive integers such that $1 \leq r_{1}<r_{2}<\cdots<r_{i}$ and let $X_{L\left(r_{1}, k\right)}, X_{L\left(r_{2}, k\right)}, \ldots, X_{L\left(r_{i}, k\right)}$ be $i$ selected GLRVs from the sequence $\left\{X_{L(n, k)}, n \geq 1\right\}$ of GLRVs of $\left\{X_{j}, j \geq 1\right\}$. Then the joint pdf of these selected GLRVs is given by

$$
\begin{aligned}
f_{X_{L\left(r_{1}, k\right)}, \ldots, X_{L\left(r_{i}, k\right)}}\left(x_{r_{1}}, \ldots, x_{r_{i}}\right) \\
=k^{r_{i}} \frac{f\left(x_{r_{1}}\right)}{F\left(x_{r_{1}}\right)} \frac{\left[-\ln F\left(x_{r_{1}}\right)\right]^{r_{1}-1}}{\Gamma\left(r_{1}\right)} \prod_{h=2}^{i} \frac{f\left(x_{r_{h}}\right)}{F\left(x_{r_{h}}\right)} \\
\quad \times \frac{\left\{\left[-\ln F\left(x_{r_{h}}\right)\right]-\left[-\ln F\left(x_{r_{h-1}}\right)\right]\right\}^{r_{h}-r_{h-1}-1}}{\Gamma\left(r_{h}-r_{h-1}\right)}\left[F\left(x_{r_{i}}\right)\right]^{k} .
\end{aligned}
$$

Proof. Let $\left\{X_{L(j, k)}, j \geq 1\right\}$ be the sequence of generalized lower(k) record values arising from an arbitrary distribution with pdf $f(x)$ and cdf $F(x)$ defined over $\mathbb{R}^{+}$. Then $\left\{Y_{j}=\frac{1}{X_{L(j, k)}}, j \geq 1\right\}$ can be considered as a sequence of generalized upper(k)record values arising from a distribution with pdf $\frac{1}{y^{2}} f\left(\frac{1}{y}\right)$ and cdf $1-F\left(\frac{1}{y}\right)$. Then the joint pdf of $Y_{1}, Y_{2}, \ldots, Y_{n}$ can be obtained from equation $(5.1)$ as

$$
\begin{aligned}
f_{Y_{1}, \ldots, Y_{n}}\left(y_{1}, \ldots, y_{n}\right) & \\
= & k^{n} \frac{\left(\frac{1}{y_{1}^{2}}\right) f\left(\frac{1}{y_{1}}\right)}{F\left(\frac{1}{y_{1}}\right)} \frac{\left(\frac{1}{y_{2}^{2}}\right) f\left(\frac{1}{y_{2}}\right)}{F\left(\frac{1}{y_{2}}\right)} \cdots \frac{\left(\frac{1}{y_{n-1}^{2}}\right) f\left(\frac{1}{y_{n-1}}\right)}{F\left(\frac{1}{y_{n-1}}\right)} \\
& \times\left(\frac{1}{y_{n}^{2}}\right) f\left(\frac{1}{y_{n}}\right)\left[F\left(\frac{1}{y_{n}}\right)\right]^{k-1},
\end{aligned}
$$

where $0<y_{1}<y_{2}<\cdots<y_{n}<\infty$. Now we derive the joint density of selected generalized upper(k)record values. From (5.3) we may write

$$
\begin{aligned}
& f_{Y_{1}, \ldots, Y_{r_{i}}}\left(y_{1}, \ldots, y_{r_{i}}\right) \\
& =k^{r_{i}} \prod_{j=1}^{r_{i}-1} \frac{\left(\frac{1}{y_{j}^{2}}\right) f\left(\frac{1}{y_{j}}\right)}{F\left(\frac{1}{y_{j}}\right)}\left(\frac{1}{y_{r_{i}}^{2}}\right) f\left(\frac{1}{y_{r_{i}}}\right)\left[F\left(\frac{1}{y_{r_{i}}}\right)\right]^{k-1} .
\end{aligned}
$$


Now if we consider the right side of (5.3) as the integrand, integrate out $y_{1}, y_{2}, \ldots$, $y_{r_{1}-1}$ over the region $0<y_{1}<\cdots<y_{r_{1}}$, integrate out $y_{r_{1}+1}, \ldots, y_{r_{2}-1}$ over the region $y_{r_{1}}<y_{r_{1}+1}<\cdots<y_{r_{2}}$ and so on and lastly integrate out $y_{r_{i-1}+1}, \ldots, y_{r_{i}-1}$ over the region $y_{r_{i-1}}<y_{r_{i-1}+1}<\cdots<y_{r_{i}}$ and simplify, then we obtain

$$
\begin{aligned}
& f_{Y_{r_{1}}, \ldots, Y_{r_{i}}}\left(y_{r_{1}}, \ldots, y_{r_{i}}\right) k^{r_{i}} \frac{\left(\frac{1}{y_{r_{1}}^{2}}\right) f\left(\frac{1}{y_{r_{1}}}\right)}{F\left(\frac{1}{y_{r_{1}}}\right)} \frac{\left[-\ln F\left(\frac{1}{y_{r_{1}}}\right)\right]^{r_{1}-1} \prod_{h=2}^{i} \frac{\left(\frac{1}{y_{r_{h}}^{2}}\right) f\left(\frac{1}{y_{r_{h}}}\right)}{\Gamma\left(\frac{1}{y_{r_{h}}}\right)}}{} \\
& \times \frac{\left\{\left[-\ln F\left(\frac{1}{y_{r_{h}}}\right)\right]-\left[-\ln F\left(\frac{1}{y_{r_{h-1}}}\right)\right]\right\}^{r_{h}-r_{h-1}-1}}{\Gamma\left(r_{h}-r_{h-1}\right)} \\
& \times\left[F\left(\frac{1}{y_{r_{i}}}\right)\right]^{k} .
\end{aligned}
$$

The above equation is the joint pdf of selected generalized upper(k)record values arising from the cdf $G(y)=1-F\left(\frac{1}{y}\right)$. Put $Z_{r_{1}}=\frac{1}{Y_{r_{1}}}, Z_{r_{2}}=\frac{1}{Y_{r_{2}}}, \ldots, Z_{r_{i}}=$ $\frac{1}{Y_{r_{i}}}$. Then the above transformation leads to a joint pdf of selected GLRVs $Z_{r_{1}}, Z_{r_{2}}, \ldots, Z_{r_{i}}$ arising from the distribution function of the reciprocal random variable of a random variable, whose cdf is $G(y)=1-F\left(\frac{1}{y}\right)$. Clearly the cdf of the random variable $Z=\frac{1}{Y}$ is $F(z)$, where the cdf of $Y$ is $G(y)$. Hence from (5.5) we obtain

$$
\begin{aligned}
f_{Z_{r_{1}}, \ldots, Z_{r_{i}}}\left(z_{r_{1}}, \ldots, z_{r_{i}}\right) \\
=k^{r_{i}} \frac{f\left(z_{r_{1}}\right)}{F\left(z_{r_{1}}\right)} \frac{\left[-\ln F\left(z_{r_{1}}\right)\right]^{z_{1}-1}}{\Gamma\left(r_{1}\right)} \prod_{h=2}^{i} \frac{f\left(z_{r_{h}}\right)}{F\left(z_{r_{h}}\right)} \\
\quad \times \frac{\left\{\left[-\ln F\left(z_{r_{h}}\right)\right]-\left[-\ln F\left(z_{r_{h-1}}\right)\right]\right\}^{r_{h}-r_{h-1}-1}}{\Gamma\left(r_{h}-r_{h-1}\right)}\left[F\left(z_{r_{i}}\right)\right]^{k}
\end{aligned}
$$

as $\left(Z_{r_{1}}, \ldots, Z_{r_{i}}\right) \stackrel{d}{=}\left(Y_{L\left(r_{1}, k\right)}, Y_{L\left(r_{2}, k\right)} \ldots Y_{L\left(r_{i}, k\right)}\right)$. The proof of the lemma follows.

Now we derive some properties of GLRVs arising from the standard FD as given below.

THEOREM 2. Let $r_{1}, r_{2}, \ldots, r_{i}$ be positive integers such that $1 \leq r_{1}<r_{2}<$ $\cdots<r_{i}$ and let $Y_{L\left(r_{1}, k\right)}, Y_{L\left(r_{2}, k\right)}, \ldots, Y_{L\left(r_{i}, k\right)}$ be the corresponding GLRVs arising from (2.3). Then the random variables $V_{1}=\frac{Y_{L\left(r_{2}, k\right)}}{Y_{L\left(r_{1}, k\right)}}, V_{2}=\frac{Y_{L\left(r_{3}, k\right)}}{Y_{L\left(r_{2}, k\right)}}, \ldots, V_{i-1}=$ $\frac{Y_{L\left(r_{i}, k\right)}}{Y_{L\left(r_{i-1}, k\right)}}$ and $V_{i}=Y_{L\left(r_{i}, k\right)}$, are all statistically independent. Further the distribution of $V_{j}$ is given by the pdf $g_{j}\left(v_{j}\right)=\frac{c v_{j}^{c r_{j}-1}\left(1-v_{j}^{c}\right)^{r_{j+1}-r_{j}-1}}{B\left(r_{j}, r_{j+1}-r_{j}\right)}, 0<v_{j}<1$, $j=1,2, \ldots, i-1$ and that of $V_{i}$ is given by the $p d f h\left(v_{i}\right)=\frac{c k^{r_{i}} e^{-k v_{i}^{-c} v_{i}^{-c r_{i}-1}}}{\Gamma\left(r_{i}\right)}$, $0<v_{i}<\infty$. 
Proof. The joint distribution of GLRVs $Y_{L\left(r_{1}, k\right)}, Y_{L\left(r_{2}, k\right)}, \ldots, Y_{L\left(r_{i}, k\right)}$ can be derived using (5.6) and is given by

$$
\begin{aligned}
f_{r_{1}, r_{2}, \ldots, r_{i}}\left(x_{r_{1}}, x_{r_{2}}, \ldots, x_{r_{i}}\right) \\
=\frac{k^{r_{i}} c^{i}}{\Gamma\left(r_{1}\right) \Gamma\left(r_{2}-r_{1}\right) \cdots \Gamma\left(r_{i}-r_{i-1}\right)} \\
\quad \times\left(x_{r_{1}}^{-c}\right)^{r_{1}-1}\left(x_{r_{2}}^{-c}-x_{r_{1}}^{-c}\right)^{r_{2}-r_{1}-1} \cdots\left(x_{r_{i}}^{-c}-x_{r_{i-1}}^{-c}\right)^{r_{i}-r_{i-1}-1} \\
\quad \times\left(x_{r_{1}} x_{r_{2}} \cdots x_{r_{i}}\right)^{-c-1} e^{-k x_{r_{i}}^{-c}}, \quad 0<x_{r_{i}}<\cdots<x_{r_{1}}<\infty .
\end{aligned}
$$

If we put $v_{1}=\frac{x_{r_{2}}}{x_{r_{1}}}, v_{2}=\frac{x_{r_{3}}}{x_{r_{2}}}, \ldots, v_{i-1}=\frac{x_{r_{i}}}{x_{r_{i-1}}}$ and $v_{i}=x_{r_{i}}$ then we have

$$
x_{r_{i}}=v_{i}, x_{r_{i-1}}=\frac{v_{i}}{v_{i-1}}, \ldots, x_{r_{2}}=\frac{v_{i}}{v_{2} v_{3} \cdots v_{i-1}}, x_{r_{1}}=\frac{v_{i}}{v_{1} v_{2} \cdots v_{i-1}} .
$$

The Jacobian of the transformation is given by

$$
|J|=\frac{v_{i}^{i-1}}{v_{1}^{2} v_{2}^{3} v_{3}^{4} \cdots v_{i-1}^{i}} .
$$

Using (5.8) in (5.7), multiplying it by $|J|$ and simplifying we get

$$
\begin{aligned}
& f\left(v_{1}, v_{2}, \ldots, v_{i}\right) \\
& =\frac{k^{r_{i}} c^{i} v_{1}^{c r_{1}-1}}{\Gamma\left(r_{1}\right) \Gamma\left(r_{2}-r_{1}\right) \cdots \Gamma\left(r_{i}-r_{i-1}\right)}\left(1-v_{1}^{c}\right)^{r_{2}-r_{1}-1} v_{2}^{c r_{2}-1}\left(1-v_{2}^{c}\right)^{r_{3}-r_{2}-1} \\
& \quad \times v_{3}^{c r_{3}-1}\left(1-v_{3}^{c}\right)^{r_{4}-r_{3}-1} \cdots v_{i-1}^{c r_{i-1}-1}\left(1-v_{i-1}^{c}\right)^{r_{i}-r_{i-1}-1} v_{i}^{-c r_{i}-1} e^{-k v_{i}^{-c}} .
\end{aligned}
$$

This proves the theorem.

We now consider the following Theorem.

THEOREM 3. Let $r_{1}, r_{2}, \ldots, r_{i}$ be positive integers such that $1 \leq r_{1}<r_{2}<$ $\cdots<r_{i}$ and let $Y_{L\left(r_{1}, k\right)}, Y_{L\left(r_{2}, k\right)}, \ldots, Y_{L\left(r_{i}, k\right)}$ be the corresponding GLRVs arising from (2.3). Then the random variables $V_{1}=\frac{Y_{L\left(r_{1}, k\right)}}{Y_{L\left(r_{2}, k\right)}}, V_{2}=\frac{Y_{L\left(r_{2}, k\right)}}{Y_{L\left(r_{3}, k\right)}}, \ldots, V_{i-1}=$ $\frac{Y_{L\left(r_{i-1}, k\right)}}{Y_{L\left(r_{i}, k\right)}}$ and $V_{i}=Y_{L\left(r_{i}, k\right)}$, are all statistically independent. Further the distribution of $V_{j}$ is given by the pdf $g_{j}\left(v_{j}\right)=\frac{c v_{j}^{-c r_{j}-1}\left(1-v_{j}^{-c}\right)^{r_{j+1}-r_{j}-1}}{B\left(r_{j}, r_{j+1}-r_{j}\right)}, v_{j}>1$, $j=1,2, \ldots, i-1$ and that of $V_{i}$ is given by the $p d f h\left(v_{i}\right)=\frac{c k^{r_{i}} e^{-k v_{i}^{-c}} v_{i}^{-c r_{i}-1}}{\Gamma\left(r_{i}\right)}$, $0<v_{i}<\infty$.

The proof of the above Theorem is similar to the proof of Theorem 2 and hence is omitted.

Corollary 5. Suppose $Y_{L(1, k)}, Y_{L(2, k)}, \ldots, Y_{L(n, k)}$ are the first $n$ GLRVs arising from the standard $F D$. Then the random variables $V_{1}=\frac{Y_{L(1, k)}}{Y_{L(2, k)}}, V_{2}=$ 
$\frac{Y_{L(2, k)}}{Y_{L(3, k)}}, \ldots, V_{n-1}=\frac{Y_{L(n-1, k)}}{Y_{L(n, k)}}$ and $V_{n}=Y_{L(n, k)}$, are all statistically independent. Further the pdf of $V_{j}$ is given by $g_{j}\left(v_{j}\right)=\frac{c v_{j}^{-c j-1}}{B(j, 1)}, v_{j}>1, j=1,2, \ldots, n-1$ and that of $V_{n}$ is given by $h\left(v_{n}\right)=\frac{c k^{n} e^{-k v_{n}^{-c} v_{n}^{-c n-1}}}{\Gamma(n)}, 0<v_{n}<\infty$.

Proof. By putting $i=n$ and $r_{1}=1, r_{2}=2, \ldots, r_{n}=n$ in Theorem 3, we get the required result.

THEOREM 4. Suppose $m$ and $n$ are positive integers such that $1 \leq m<n$ and $Y_{L(m, k)}$ and $Y_{L(n, k)}$ are $m$ th and $n$th $G L R V$ arising from the standard $F D$ defined in (2.3). Then

$$
E\left\{\frac{Y_{L(m, k)}}{Y_{L(n, k)}}\right\}=\frac{E\left(Y_{L(m, k)}\right)}{E\left(Y_{L(n, k)}\right)} .
$$

Proof. We have

$$
E\left(Y_{L(m, k)}\right)=E\left(\left\{\frac{Y_{L(m, k)}}{Y_{L(n, k)}}\right\} Y_{L(n, k)}\right) .
$$

If we put $i=2, r_{1}=m$ and $r_{2}=n$ in Theorem 3, we observe the independence of $\frac{Y_{L(m, k)}}{Y_{L(n, k)}}$ and $Y_{L(n, k)}$ and hence we have

$$
E\left(Y_{L(m, k)}\right)=E\left\{\frac{Y_{L(m, k)}}{Y_{L(n, k)}}\right\} E\left(Y_{L(n, k)}\right) .
$$

The required result then easily follows.

As a consequence of the above Theorem we then have

$$
E\left\{\frac{Y_{L(m, k)}}{Y_{L(n, k)}}\right\}=\frac{\Gamma(n) \Gamma\left(m-\frac{1}{c}\right)}{\Gamma(m) \Gamma\left(n-\frac{1}{c}\right)}, \quad \text { provided } m>\frac{1}{c} .
$$

The importance of Theorem 3 is that it helps one to obtain the product moments of any order of GLRVs arising from the standard the FD in a very simple and elegant manner. For example if $p, r, s, t$ are positive integers such that $1 \leq$ $p<r<s<t$, then for the GLRVs $Y_{L(p, k)}, Y_{L(r, k)}, Y_{L(s, k)}, Y_{L(t, k)}$ and positive integers $a, b, d, e$ we have

$$
\begin{aligned}
E\left[Y_{L(p, k)}^{a} Y_{L(r, k)}^{b} Y_{L(s, k)}^{d} Y_{L(t, k)}^{e}\right]=E & {\left[\left\{\frac{Y_{L(p, k)}}{Y_{L(r, k)}}\right\}^{a}\left\{\frac{Y_{L(r, k)}}{Y_{L(s, k)}}\right\}^{a+b}\right.} \\
& \left.\times\left\{\frac{Y_{L(s, k)}}{Y_{L(t, k)}}\right\}^{a+b+d}\left\{Y_{L(t, k)}\right\}^{a+b+d+e}\right] .
\end{aligned}
$$


If we put $V_{1}=\frac{Y_{L(p, k)}}{Y_{L(r, k)}}, V_{2}=\frac{Y_{L(r, k)}}{Y_{L(s, k)}}, V_{3}=\frac{Y_{L(s, k)}}{Y_{L(t, k)}}$ and $V_{4}=Y_{L(t, k)}$, then from Theorem 3, we have

$$
\begin{aligned}
E\left[Y_{L(p, k)}^{a} Y_{L(r, k)}^{b} Y_{L(s, k)}^{d} Y_{L(t, k)}^{e}\right] & =E\left[\left\{V_{1}\right\}^{a}\left\{V_{2}\right\}^{a+b}\left\{V_{3}\right\}^{a+b+d}\left\{V_{4}\right\}^{a+b+d+e}\right] \\
= & E\left[\left\{V_{1}\right\}^{a}\right] E\left[\left\{V_{2}\right\}^{a+b}\right] E\left[\left\{V_{3}\right\}^{a+b+d}\right] E\left[\left\{V_{4}\right\}^{a+b+d+e}\right] \\
= & \frac{B\left(p-\frac{a}{c}, r-p\right)}{B(p, r-p)} \times \frac{B\left(r-\frac{a}{c}-\frac{b}{c}, s-r\right)}{B(r, s-r)} \\
& \times \frac{B\left(s-\frac{a}{c}-\frac{b}{c}-\frac{d}{c}, t-s\right)}{B(s, t-s)} \times \frac{k^{(a+b+d+e) / c} \Gamma\left(t-\frac{a}{c}-\frac{b}{c}-\frac{d}{c}-\frac{e}{c}\right)}{\Gamma(t)} \\
& \quad \Gamma\left(p-\frac{a}{c}\right) \Gamma\left(r-\frac{a+b}{c}\right) \Gamma\left(s-\frac{a+b+d}{c}\right) \Gamma\left(t-\frac{a+b+d+e}{c}\right) \\
= & \frac{\Gamma(p) \Gamma\left(r-\frac{a}{c}\right) \Gamma\left(s-\frac{a+b}{c}\right) \Gamma\left(t-\frac{a+b+d}{c}\right)}{c}
\end{aligned}
$$

provided $p>\frac{a}{c}, r>\frac{a+b}{c}, s>\frac{a+b+d}{c}$ and $t>\frac{a+b+d+e}{c}$.

\section{Estimation of the parameters of the FD}

In this section we consider the situations where the experimental details available are about only GLRVs observed at various time points on the variable under study. Hence the estimation methods proposed in both Subsections 6.1 and 6.2 utilize only the available GLRVs.

\subsection{Estimation of the parameters of the FD when the shape param- eter $c$ is known}

In this section we describe the problem of the estimation of the location parameter $\theta$ and scale parameter $\sigma$ of the FD defined by (2.1) based on the available GLRVs provided the shape parameter $c$ is known by the least-squares method. Let $X_{L(1, k)}, X_{L(2, k)}, \ldots, X_{L(n, k)}$ be the first $n$ GLRVs arising from the FD defined in (2.1) with known $c$. Then clearly $Y_{L(i, k)}=\left(X_{L(i, k)}-\theta\right) / \sigma, i=$ $1,2, \ldots, n$ are distributed as the first $n$ GLRVs arising from the standard FD defined in (2.3). Assume that the mean and variance of $Y_{L(i, k)}$ are finite for $1 \leq i \leq n$. From (3.3) and (3.5) we write $\alpha_{i}=E\left(Y_{L(i, k)}\right)=k^{1 / c} \frac{\Gamma\left(i-\frac{1}{c}\right)}{\Gamma(i)}$ and $\sigma_{i, j}=\operatorname{Cov}\left(Y_{L(i, k)}, Y_{L(j, k)}\right)=\frac{k^{2 / c} \Gamma\left(i-\frac{1}{c}\right)}{\Gamma(i)}\left\{\frac{\Gamma\left(j-\frac{2}{c}\right)}{\Gamma\left(j-\frac{1}{c}\right)}-\frac{\Gamma\left(j-\frac{1}{c}\right)}{\Gamma(j)}\right\}$.

Let $\boldsymbol{X}=\left[X_{L(1, k)}, X_{L(2, k)}, \ldots, X_{L(n, k)}\right]^{T}$ and $\boldsymbol{Y}=\left[Y_{L(1, k)}, Y_{L(2, k)}, \ldots\right.$, $\left.Y_{L(n, k)}\right]^{T}$. Then the mean vector $E(\boldsymbol{Y})$ and the dispersion matrix $D(\boldsymbol{Y})$ of $\boldsymbol{Y}$ are given by $E(\boldsymbol{Y})=\boldsymbol{\alpha}=\left[\alpha_{1}, \alpha_{2}, \ldots, \alpha_{n}\right]^{T}$ and $D(\boldsymbol{Y})=\boldsymbol{\Sigma}=\left(\left(\sigma_{i, j}\right)\right)$. Now we can write

$$
E(X)=1 \theta+\alpha \sigma
$$

where $\mathbf{1}$ is a column vector of $n$ ones and

$$
D(\boldsymbol{X})=\sigma^{2} \boldsymbol{\Sigma} .
$$


Clearly (6.1) and (6.2) form the well-known generalized Gauss-Markoff setup. Then for

$$
\boldsymbol{\Delta}=\left(\boldsymbol{\alpha}^{T} \boldsymbol{\Sigma}^{-1} \boldsymbol{\alpha}\right)\left(\mathbf{1}^{T} \boldsymbol{\Sigma}^{-1} \mathbf{1}\right)-\left(\boldsymbol{\alpha}^{T} \boldsymbol{\Sigma}^{-1} \mathbf{1}\right)^{2},
$$

we obtain the BLUEs $\theta^{*}$ and $\sigma^{*}$ of $\theta$ and $\sigma$ respectively as (see, Arnold et al. (1998))

$$
\theta^{*}=\left[\frac{\boldsymbol{\alpha}^{T} \boldsymbol{\Sigma}^{-1}\left(\boldsymbol{\alpha} \mathbf{1}^{T}-\mathbf{1} \boldsymbol{\alpha}^{T}\right) \boldsymbol{\Sigma}^{-1}}{\boldsymbol{\Delta}}\right] \boldsymbol{X}=\sum_{j=1}^{n} a_{j: n} X_{L(j, k)}=\boldsymbol{a}^{T} \boldsymbol{X}
$$

and

$$
\sigma^{*}=\left[\frac{\mathbf{1}^{T} \boldsymbol{\Sigma}^{-1}\left(\mathbf{1} \boldsymbol{\alpha}^{T}-\boldsymbol{\alpha} \mathbf{1}^{T}\right) \boldsymbol{\Sigma}^{-1}}{\boldsymbol{\Delta}}\right] \boldsymbol{X}=\sum_{j=1}^{n} b_{j: n} X_{L(j, k)}=\boldsymbol{b}^{T} \boldsymbol{X}
$$

where $\boldsymbol{a}=\left(a_{1: n}, a_{2: n}, a_{3: n}, \ldots, a_{n: n}\right)^{T}$ and $\boldsymbol{b}=\left(b_{1: n}, b_{2: n}, b_{3: n}, \ldots, b_{n: n}\right)^{T}$ are the vectors of constants. Further

$$
\begin{aligned}
& \operatorname{Var}\left(\theta^{*}\right)=\sigma^{2}\left\{\frac{\boldsymbol{\alpha}^{T} \boldsymbol{\Sigma}^{-1} \boldsymbol{\alpha}}{\boldsymbol{\Delta}}\right\}, \quad \operatorname{Var}\left(\sigma^{*}\right)=\sigma^{2}\left\{\frac{\mathbf{1}^{T} \boldsymbol{\Sigma}^{-1} \mathbf{1}}{\boldsymbol{\Delta}}\right\} \quad \text { and } \\
& \operatorname{Cov}\left(\theta^{*}, \sigma^{*}\right)=\sigma^{2}\left\{\frac{-\boldsymbol{\alpha}^{T} \boldsymbol{\Sigma}^{-1} \mathbf{1}}{\boldsymbol{\Delta}}\right\} .
\end{aligned}
$$

Now based on the expectations, variances and covariances of GLRVs arising from the FD we state and prove the following theorem, which helps one to obtain the coefficients of GLRVs involved in the estimators of $\theta$ and $\sigma$ of the FD from the corresponding coefficients of the classical lower record values involved in the estimates of the above parameters.

THEOREM 5. Let $\left\{X_{n}, n \geq 1\right\}$ be a sequence of iid random variables each distributed identically as a FD with pdf $f(x)$ given in (2.1). Let $\left\{X_{L(n, k)}\right\}$ be the sequence of GLRVs generated from the sequence $\left\{X_{n}\right\}$. Then the coefficient vectors $\boldsymbol{a}$ and $\boldsymbol{b}$ of the vectors of the vector of the first $n$ GLRVs involved in the Best Linear Unbiased Estimates $\theta^{*}$ and $\sigma^{*}$ of the location parameter $(\theta)$ and the scale parameter $(\sigma)$ respectively are given by

$$
\boldsymbol{a}=\boldsymbol{a}^{*} \quad \text { and } \quad \boldsymbol{b}=k^{-1 / c} \boldsymbol{b}^{*},
$$

where $\boldsymbol{a}^{*}$ and $\boldsymbol{b}^{*}$ are the coefficient vectors of the first $n$ classical lower record values involved in the Best Linear Unbiased Estimates $\theta_{0}^{*}$ and $\sigma_{0}^{*}$ of the location and scale parameters of the FD respectively. Further

$$
\begin{aligned}
& \operatorname{Var}\left(\theta^{*}\right)=k^{2 / c} \operatorname{Var}\left(\theta_{0}^{*}\right), \quad \operatorname{Var}\left(\sigma^{*}\right)=\operatorname{Var}\left(\sigma_{0}^{*}\right) \quad \text { and } \\
& \operatorname{Cov}\left(\theta^{*}, \sigma^{*}\right)=k^{1 / c} \operatorname{Cov}\left(\theta_{0}^{*}, \sigma_{0}^{*}\right) .
\end{aligned}
$$


Proof. Let $\boldsymbol{a}$ be the coefficient vector of the BLUE of the location parameter $(\theta)$ based on the first $n$ GLRVs. Then from (6.3) we have

$$
\boldsymbol{a}=\left[\frac{\boldsymbol{\alpha}^{T} \boldsymbol{\Sigma}^{-1}\left(\boldsymbol{\alpha} \mathbf{1}^{T}-\mathbf{1} \boldsymbol{\alpha}^{T}\right) \boldsymbol{\Sigma}^{-1}}{\left(\boldsymbol{\alpha}^{T} \boldsymbol{\Sigma}^{-1} \boldsymbol{\alpha}\right)\left(\mathbf{1}^{T} \boldsymbol{\Sigma}^{-1} \mathbf{1}\right)-\left(\boldsymbol{\alpha}^{T} \boldsymbol{\Sigma}^{-1} \mathbf{1}\right)^{2}}\right] .
$$

From (3.3), (3.4) and (3.5) we can write

$$
\boldsymbol{\alpha}=k^{1 / c} \boldsymbol{\alpha}_{0} \quad \text { and } \quad \boldsymbol{\Sigma}^{-1}=k^{-2 / c} \boldsymbol{\Sigma}_{0}^{-1},
$$

where $\boldsymbol{\alpha}_{0}$ and $\boldsymbol{\Sigma}_{0}^{-1}$ are the mean vector and inverse of the dispersion matrix of the vector of the first $n$ classical lower record values arising from the standard FD. So the coefficient vector of the BLUE of the location parameter based on first $n$ GLRVs can be simplified as

$$
\begin{aligned}
\boldsymbol{a} & =\left[\frac{k^{1 / c} \boldsymbol{\alpha}_{0}^{T} k^{-2 / c} \boldsymbol{\Sigma}_{0}^{-1}\left(k^{1 / c} \boldsymbol{\alpha}_{0} \mathbf{1}^{T}-\mathbf{1} k^{1 / c} \boldsymbol{\alpha}_{0}^{T}\right) k^{-2 / c} \boldsymbol{\Sigma}_{0}^{-1}}{\left(k^{1 / c} \boldsymbol{\alpha}_{0}^{T} k^{-2 / c} \boldsymbol{\Sigma}_{0}^{-1} k^{1 / c} \boldsymbol{\alpha}_{0}\right)\left(\mathbf{1}^{T} k^{-2 / c} \boldsymbol{\Sigma}_{0}^{-1} \mathbf{1}\right)-\left(k^{1 / c} \boldsymbol{\alpha}_{0}^{T} k^{-2 / c} \boldsymbol{\Sigma}_{0}^{-1} \mathbf{1}\right)^{2}}\right] \\
& =\left[\frac{\boldsymbol{\alpha}_{0}^{T} \boldsymbol{\Sigma}_{0}^{-1}\left(\boldsymbol{\alpha}_{0} \mathbf{1}^{T}-\mathbf{1} \boldsymbol{\alpha}_{0}^{T}\right) \boldsymbol{\Sigma}_{0}^{-1}}{\left(\boldsymbol{\alpha}_{0}^{T} \boldsymbol{\Sigma}_{0}^{-1} \boldsymbol{\alpha}_{0}\right)\left(\mathbf{1}^{T} \boldsymbol{\Sigma}_{0}^{-1} \mathbf{1}\right)-\left(\boldsymbol{\alpha}_{0}^{T} \boldsymbol{\Sigma}_{0}^{-1} \mathbf{1}\right)^{2}}\right]=\boldsymbol{a}^{*}
\end{aligned}
$$

The proof of $\boldsymbol{b}=k^{-1 / c} \boldsymbol{b}^{*}, \operatorname{Var}\left(\theta^{*}\right)=k^{2 / c} \operatorname{Var}\left(\theta_{0}^{*}\right), \operatorname{Var}\left(\sigma^{*}\right)=\operatorname{Var}\left(\sigma_{0}^{*}\right)$ and $\operatorname{Cov}\left(\theta^{*}, \sigma^{*}\right)=k^{1 / c} \operatorname{Cov}\left(\theta_{0}^{*}, \sigma_{0}^{*}\right)$, follows easily, if we proceed as in the case of proving $\boldsymbol{a}=\boldsymbol{a}^{*}$.

Remark 1 . From the results of Theorem 5 we conclude that the estimate $\theta^{*}$ of $\theta$ is lower in precision than the estimate $\theta_{0}^{*}$. However $\sigma$ is estimated with equal precision by $\sigma^{*}$ and $\sigma_{0}^{*}$ for all values of $c$.

Sulthan (2010) has tabulated the component values of the vectors $\boldsymbol{a}^{*}, \boldsymbol{b}^{*}$, $\operatorname{Var}\left(\theta_{0}^{*}\right)$ and $\operatorname{Var}\left(\sigma_{0}^{*}\right)$ for different values of $n$. We can use those tabulated values and the results of Theorem 5 , to obtain the coefficient vectors $\boldsymbol{a}$ and $\boldsymbol{b}$ of $\theta^{*}$ and $\sigma^{*}$ respectively and their variances without any direct computation for determining them.

\subsection{Estimation of the parameters of the FD when all parameters are unknown}

It is unrealistic to assume that the shape parameter $c$ of the FD is always known. However when $c$ is unknown we may first recommend an estimator of $c$ and then use the BLUEs $\theta^{*}$ and $\sigma^{*}$ for estimating $\theta$ and $\sigma$. In this case we make use of a systematic approach utilizing the concept of the method of moments estimation and BLUE using GLRVs to estimate the parameters. If $X_{L(1, k)}, X_{L(2, k)}, \ldots, X_{L(n, k)}$ are the first $n$ available GLRVs arising from (2.1), then it is clear to note that $\theta \leq X_{L(n, k)}<X_{L(n-1, k)}<\cdots<X_{L(1, k)}<\infty$. As $X_{L(n, k)}$ is the closest observation point to $\theta$ we may take $X_{L(n, k)}=\theta_{0}$ as an 
estimate of $\theta$. Now from (5.11), if $i>\frac{1}{c}$ then we can write

$$
E\left\{\frac{X_{L(i, k)}-\theta}{X_{L(i+1, k)}-\theta}\right\}=\frac{\Gamma(i+1) \Gamma\left(i-\frac{1}{c}\right)}{\Gamma(i) \Gamma\left(i+1-\frac{1}{c}\right)}=\frac{i}{i-\frac{1}{c}}
$$

as $\frac{X_{L(i, k)}-\theta}{X_{L(i+1, k)}-\theta}$ which is distributed identically as the ratio, $\frac{Y_{L(i, k)}}{Y_{L(i+1, k)}}$ of GLRVs arising from the standard FD defined by the pdf $f_{0}(y)$ which is given in $(2.3)$. Now keeping in mind the approach adopted in the method of moments estimation we write the following

$$
\frac{X_{L(i, k)}-\theta_{0}}{X_{L(i+1, k)}-\theta_{0}}=\frac{i}{i-\frac{1}{c}}, \quad i=1,2, \ldots, n-1 .
$$

Then an estimator $\hat{c}_{i}$ of $c$ based on $X_{L(i, k)}, X_{L(i+1, k)}$ and $\theta_{0}=X_{L(n, k)}$ is given by

$$
\hat{c}_{i}=\frac{X_{L(i, k)}-\theta_{0}}{i\left(X_{L(i, k)}-X_{L(i+1, k)}\right)}, \quad i=1,2, \ldots, n-1
$$

Now there are $n-1$ estimates $\hat{c}_{1}, \hat{c}_{2}, \ldots, \hat{c}_{n-1}$ for $c$ so that, we may take the mean of these estimates as an estimate $\hat{c}$ of $c$ and is given by

$$
\hat{c}=\frac{\sum_{i=1}^{n-1} \hat{c}_{i}}{n-1}
$$

Using this as a known value of $c$, now we use the results of Subsection 6.1 to estimate ultimately $\theta$ and $\sigma$ by BLUEs.

To illustrate the closeness of the estimated value of $c$ by using (6.9) with its true value, we have used Mathematica software and simulated 100 independent observations from the FD with scale parameter $\sigma=2.0$ and location parameter $\mu=1.0$ and for some assumed values of $c$, collected GLRVs from them and repeated it for 1000 runs. In each case we estimated the shape parameter $c$, taken its average and it is given below.

It seems that in each case the estimated value $\hat{c}$ is very close to the true value of the parameter $c$.

Table 2. Estimates of $c$ for various values of $k$ based on a simulation study with 1000 runs, with $\mu=1.0, \sigma=2.0, c=2.5(0.5) 3.5$ and $k=2(1) 4$.

\begin{tabular}{|c|c|c|c|c|c|c|c|c|c|}
\hline & \multicolumn{3}{|c|}{$c=2.5$} & \multicolumn{3}{c|}{$c=3$} & \multicolumn{3}{c|}{$c=3.5$} \\
\hline & $k=2$ & $k=3$ & $k=4$ & $k=2$ & $k=3$ & $k=4$ & $k=2$ & $k=3$ & $k=4$ \\
\hline$\hat{c}$ & 2.5103 & 2.5080 & 2.5005 & 3.0136 & 3.0117 & 3.0046 & 3.5295 & 3.5172 & 3.50126 \\
\hline
\end{tabular}




\section{Prediction of future records using BLUP}

Prediction of future records are of natural interest in many contexts. Statistical prediction is the problem of inferring the values of unknown future variables or functions of such variables from current available informative observations. In the available literature one can observe that considerable works have been carried out on both parametric and nonparametric predictions. For more details on prediction one may refer to Gulati and Padgett (2003).

Now we discuss the problem of predicting the future GLRV using the available GLRVs of the FD for known values of $c$. Suppose $X_{L(1, k)}, X_{L(2, k)}, \ldots, X_{L(n, k)}$ are the $n$ available GLRVs. Then our interest here is on predicting the next GLRV $X_{L(n+1, k)}$. The best linear unbiased predicted value of the next record can be obtained (see, Arnold et al. (1998)) as

$$
X_{L(n+1, k)}^{*}=\theta^{*}+\alpha_{n+1} \sigma^{*}+\boldsymbol{w}^{T} \Sigma^{-1}\left(\boldsymbol{X}-\theta^{*} \mathbf{1}-\sigma^{*} \boldsymbol{\alpha}\right),
$$

where $\boldsymbol{X}$ is the vector of the $n$ observed GLRVs from the FD as defined in (2.1), $\boldsymbol{\alpha}$ and $\boldsymbol{\Sigma}$ are the vector of means and dispersion matrix of the vector of $n$ GLRVs arising from the standard FD, $\mathbf{1}$ is a vector of $n$ one's, $\alpha_{n+1}$ is the expected value of $(n+1)$ th GLRV arising from the standard FD and $\boldsymbol{w}^{T}=$ $\left(\sigma_{1, n+1}, \sigma_{2, n+1}, \ldots, \sigma_{n, n+1}\right)$. The equation $(7.1)$ can also be written as

$$
X_{L(n+1, k)}^{*}=\sum_{i=1}^{n} f_{i: n} X_{L(i, k)}=\boldsymbol{f}^{T} \boldsymbol{X}
$$

where the coefficient vector $\boldsymbol{f}$ of constants of $X$ is $\boldsymbol{f}=\left(f_{1: n}, f_{2: n}, \ldots, f_{n: n}\right)^{T}$. Now we prove the following Theorem.

THEOREM 6. Let $X_{L(1, k)}, X_{L(2, k)}, \ldots, X_{L(n, k)}$ be the first $n$ available GLRVs and let $X_{L(1,1)}, X_{L(2,1)}, \ldots, X_{L(n, 1)}$ be the first $n$ classical lower record values. Then the coefficients $f_{i: n}$ of $X_{L(i, k)}, i=1,2, \ldots, n$ involved in the BLUP of the next GLRV $X_{L(n+1, k)}$ as given in $X_{L(n+1, k)}^{*}=\sum_{i=1}^{n} f_{i: n} X_{L(i, k)}$ are identically the same as the coefficients of the classical lower record values $X_{L(i, 1)}, i=1,2, \ldots, n$ involved in the BLUP of the next classical lower record value $X_{L(n+1,1)}$. That is

$$
f=f^{*}
$$

where $\boldsymbol{f}^{*}$ is the coefficient vector in the BLUP of $X_{L(n+1,1)}$ based on the first $n$ classical lower record values.

Proof. If the vectors $\boldsymbol{a}$ and $\boldsymbol{b}$ are as defined in (6.3) and (6.4) respectively then the best linear unbiased predicted value of the next GLRV can be obtained by the equation

$$
\begin{aligned}
X_{L(n+1, k)}^{*} & =\theta^{*}+\alpha_{n+1} \sigma^{*}+\boldsymbol{w}^{T} \Sigma^{-1}\left(\boldsymbol{X}-\theta^{*} \mathbf{1}-\sigma^{*} \boldsymbol{\alpha}\right) \\
& =\boldsymbol{a}^{T} \boldsymbol{X}+\alpha_{n+1} \boldsymbol{b}^{T} \boldsymbol{X}+\boldsymbol{w}^{T} \Sigma^{-1}\left(\boldsymbol{X}-\left(\boldsymbol{a}^{T} \boldsymbol{X}\right) \mathbf{1}-\left(\boldsymbol{b}^{T} \boldsymbol{X}\right) \boldsymbol{\alpha}\right) \\
& =\left[\boldsymbol{a}^{T}+\alpha_{n+1} \boldsymbol{b}^{T}+\boldsymbol{w}^{T} \Sigma^{-1}\left(\mathbf{1}-\boldsymbol{a}^{T} \mathbf{1}-\boldsymbol{b}^{T} \boldsymbol{\alpha}\right)\right] \boldsymbol{X}
\end{aligned}
$$




$$
=\boldsymbol{f}^{T} \boldsymbol{X}
$$

Now using the relation between $\boldsymbol{a}$ and $\boldsymbol{a}^{*}$ and that between $\boldsymbol{b}$ and $\boldsymbol{b}^{*}$ as given in (6.6)

$$
\begin{aligned}
(7.5) X_{L(n+1, k)}^{*}= & {\left[\left(\boldsymbol{a}^{*}\right)^{T}+k^{1 / c} \alpha_{0(n+1)}\left(\frac{1}{k^{1 / c}} \boldsymbol{b}^{*}\right)^{T}+k^{2 / c} \boldsymbol{w}_{0}^{T} k^{-2 / c} \Sigma_{0}^{-1}\right.} \\
& \left.\times\left\{\boldsymbol{1}-\left(\boldsymbol{a}^{*}\right)^{T} \mathbf{1}-\left(\frac{1}{k^{1 / c}} \boldsymbol{b}^{*}\right)^{T} k^{1 / c} \boldsymbol{\alpha}_{0}\right\}\right] \boldsymbol{X} \\
& =\left[\left(\boldsymbol{a}^{*}\right)^{T}+\alpha_{0(n+1)}\left(\boldsymbol{b}^{*}\right)^{T}+\boldsymbol{w}_{0}^{T} \Sigma_{0}^{-1}\left\{\mathbf{1}-\left(\boldsymbol{a}^{*}\right)^{T} \mathbf{1}-\left(\boldsymbol{b}^{*}\right)^{T} \boldsymbol{\alpha}_{0}\right\}\right] \boldsymbol{X} \\
& =\left(\boldsymbol{f}^{*}\right)^{T} \boldsymbol{X} .
\end{aligned}
$$

From (7.4) and (7.5), the proof of the Theorem follows.

The prediction coefficients of the available record values or GLRVs in predicting a future record is not seen determined specifically for the FD and hence

Table 3. Coefficients $f_{i: n}$ of $X_{L(i, k)}$ involved in the BLUP $X_{L(n+1, k)}^{*}=\sum_{i=1}^{n} f_{i: n}\left(X_{L(i, k)}\right)$ for $n=2(1) 5, c=2.5(0.5) 5$ and for any positive integer $k \geq 1$.

\begin{tabular}{|l|l|l|r|r|r|l|}
\hline \multicolumn{2}{|c|}{} & \multicolumn{5}{|c|}{ Coefficients $f_{i: n}$ of $X_{L(i, k)}$} \\
\hline$n$ & \multicolumn{1}{|c|}{$c$} & $f_{n: 1}$ & $f_{n: 2}$ & $f_{n: 3}$ & $f_{n: 4}$ & $f_{n: 5}$ \\
\hline 2 & 2.5 & -0.30000 & 1.30000 & & & \\
& 3 & -0.33333 & 1.33333 & & & \\
& 3.5 & -0.56123 & 1.56123 & & & \\
& 4 & -0.37500 & 1.37500 & & & \\
& 4.5 & -0.38889 & 1.38889 & & & \\
& 5 & -0.40000 & 1.40000 & & & \\
3 & 2.5 & -0.03334 & -0.38889 & 1.42223 & & \\
& 3 & -0.05555 & -0.33334 & 1.38889 & & \\
& 3.5 & -0.07143 & -0.30001 & 1.37144 & & \\
& 4 & -0.08334 & -0.27775 & 1.36109 & & \\
& 4.5 & -0.09258 & -0.26194 & 1.35452 & & \\
& 5 & -0.09999 & -0.25003 & 1.35002 & & \\
4 & 2.5 & -0.01047 & -0.12210 & -0.20345 & 1.33602 & \\
& 3 & -0.01960 & -0.11764 & -0.17653 & 1.31377 & \\
& 3.5 & -0.02706 & -0.11362 & -0.15911 & 1.29979 & \\
& 4 & -0.03309 & -0.11030 & -0.14704 & 1.29043 & \\
& 4.5 & -0.03801 & -0.10756 & -0.13820 & 1.28377 & \\
& 5 & -0.04213 & -0.10535 & -0.13118 & 1.27866 & \\
5 & 2.5 & -0.00467 & -0.05448 & -0.09081 & -0.12383 & 1.27379 \\
& 3 & -0.00933 & -0.05601 & -0.08402 & -0.10789 & 1.25725 \\
& 3.5 & -0.01343 & -0.05640 & -0.07912 & -0.09731 & 1.24626 \\
& 4 & -0.01692 & -0.05640 & -0.07498 & -0.09063 & 1.23893 \\
& 4.5 & -0.01986 & -0.05615 & -0.07236 & -0.08468 & 1.23305 \\
& 5 & -0.02235 & -0.05591 & -0.06999 & -0.08055 & 1.22880 \\
\hline
\end{tabular}


we have determined those values for $i=1,2, \ldots, n, n=2(1) 5, c=2.5(0.5) 5$ and they are presented in Table 3. Due to the invariance nature of these coefficients for changing values of $k$, the above coefficients can be used for prediction of a future classical lower record value or for the prediction of a future GLRV for any $k \geq 2$ as well.

If $c$ involved in (2.1) is unknown, then we use the estimate $\hat{c}$ as given in (6.9) and use that as a known value of $c$ so as to apply the procedure described above to predict the future record value. As an illustration of the application of the results derived in this section, now we consider the GLRVs arising from the data given in Table 1, which may be considered as a sample arising from the FD. When $k=2$, the number of generalized records observed is 12 and are given by: $164.02,145.02,115.02,112.78,110.29,109.81,107.44,107.25,106.7,105.35$, 104.71, 103.14. We have estimated the value of $c$ based on these records and by the equation (6.9) as 1.36205. The condition for the existence of moments of GLRV with this estimated value of $c$ will be satisfied for $n \geq 2$. So we consider only the GLRVs $X_{L(i, 2)}$, for $i=2,3, \ldots, 12$ for further estimation procedures by treating the data as left censored GLRVs excluding $X_{L(1, k)}$. We have evaluated the parameters $\theta$ and $\sigma$ as suggested in Subsection 6.1 and the obtained estimates are $\theta^{*}=93.8519$ and $\sigma^{*}=32.762$. Further we use the formula given in (7.1) to predict the 13 th generalized(2)lower record value and it is estimated as 102.572 .

\section{Acknowledgements}

The authors express their gratefulness for many of the constructive comments of the learned referees which helped to improve considerably the revised version of the paper. The first author acknowledges his gratefulness to the University Grants Commission, Government of India for providing financial assistance in the form of UGC-Emeritus Fellowship. The second author is also very grateful to the University Grants Commission, Government of India for the financial support received for this research work in the form of MAN-JRF.

\section{REFERENCES}

Aczel, J. (1966). Lectures on Functional Equations and Their Applications, Academic Press, New York.

Ahmadi, J., Jozani, M. J., Marchand, E. and Parsian, A. (2009). Bayes estimation based on $k$-record data from a general class of distributions under balanced type loss functions, $J$. Statist. Plann. Inference, 139, 1180-1189.

Ahsanullah, M. (1998). Record Values: Theory and Applications, University Press of America Inc., Lanham, Maryland, USA.

Arnold, B. C., Balakrishnan, N. and Nagaraja, H. N. (1998). Records, John Wiley and Sons, New York.

Bieniek, M. and Szynal, D. (2007). On $k$ th record times, record values and their moments, $J$. Statist. Plann. Inference, 137, 12-22.

Bury, K. (1999). Statistical Distributions in Engineering, Cambridge University Press.

Castillo, E., Hadi, A. S., Balakrishnan, N. and Sarabia, J. M. (2004). Extreme Value and Related Models with Applications in Engineering and Science, John Wiley and Sons, New York.

Chandler, K. N. (1952). The distribution and frequency of record values, J. Roy. Statist. Soc. Ser. $B, \mathbf{1 4}, 220-228$. 
Coles, S., Bawa, J., Trenner, L. and Dorazio, P. (2001). An Introduction to Statistical Modeling of Extreme Values, Vol. 208, London, Springer.

Dziubdziela, W. and Kopocinski, B. (1976). Limiting properties of the $k$ th record values, Appl. Math., 15, 187-190.

Fréchet, M. (1927). Sur la loi de probabilite de lecart maximum, Ann. Soc. Polon. Math. Cracovie, 6, 93-116.

Gulati, S. and Padgett, W. J. (2003). Parametric and Nonparametric Inference from RecordBreaking Data, Springer-Verlag, New York.

Minimol, S. and Thomas, P. Y. (2013). On some properties of Makeham distribution using generalized record values and its characterizations, Brazilian J. Probability and Statistics, 27, 487-501.

Minimol, S. and Thomas, P. Y. (2014). On characterization of Gompertz distribution by generalized record values, Journal of Statistical Theory and Applications, 13, 38-45.

Nevzorov, V. (2001). Records: Mathematical Theory, Translation of Mathematical Monographs, vol. 194, American Mathematical Society, Providence, RI, USA.

Pawlas, P. and Szynal, D. (1998). Relations for single and product moment of $k$ th record values from exponential and Gumbel distribution, J. Appl. Statist. Sci., 7, 53-62.

Pawlas, P. and Szynal, D. (1999). Recurrence relations for single and product moment of $k$ th record values from Pareto, generalized Pareto and Burr distributions, Comm. Statist.Theory Methods, 28, 1699-1709.

Pawlas, P. and Szynal, D. (2000). Characterizations of the inverse Weibull distribution and generalized extreme value distributions by moments of $k$ th record values, Applicationes Mathematicae, 27, 197-202.

Stam, A. J. (1985). Independent Poisson processes generated by record values and inter-record times, Stochastic Processes and their Applications, 19, 315-325.

Sulthan, K. S. (2010). Record values from the inverse Weibull lifetime model: different methods of estimation, Intelligent Information Management, 2, 61-66. 